\title{
Patrinia scabiosaefolia inhibits colorectal cancer growth through suppression of tumor angiogenesis
}

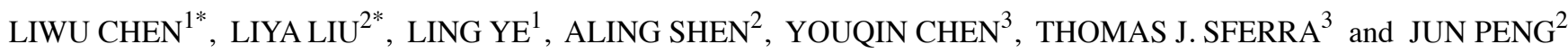 \\ ${ }^{1}$ Department of Surgery, Second Affiliated Hospital of Fujian University of Traditional Chinese Medicine, \\ Fuzhou, Fujian 350003; ${ }^{2}$ Academy of Integrative Medicine, Fujian University of Traditional Chinese Medicine, \\ Fuzhou, Fujian 350122, P.R. China; ${ }^{3}$ Rainbow Babies and Children's Hospital, \\ Case Western Reserve University School of Medicine, Cleveland, OH 44106, USA
}

Received April 16, 2013; Accepted June 14, 2013

DOI: $10.3892 /$ or.2013.2582

\begin{abstract}
Angiogenesis is an essential process for tumor development and metastasis, therefore inhibition of tumor angiogenesis has become a promising strategy for anticancer treatments. Patrinia scabiosaefolia, a well-known Oriental folk medicine, has been shown to be effective in the clinical treatment of gastrointestinal cancers. However, the precise mechanism of its tumoricidal activity remains largely unknown. Using a colorectal cancer (CRC) mouse xenograft model, the human colon carcinoma cell line HT-29 and human umbilical vein endothelial cells (HUVECs), in the present study we evaluated the effects of an ethanol extract of Patrinia scabiosaefolia (EEPS) on tumor angiogenesis in vivo and in vitro, and investigated the underlying molecular mechanisms. We found that EEPS treatment significantly reduced the tumor volume in CRC mice and decreased the intratumoral microvessel density in tumor tissues. In addition, EEPS inhibited several key processes of angiogenesis, including the proliferation, migration and tube formation of HUVECs. Moreover, EEPS treatment suppressed the expression of VEGF-A in CRC tumors and HT-29 cells. Collectively, our data suggest that Patrinia scabiosaefolia inhibits CRC growth likely via suppression of tumor angiogenesis.
\end{abstract}

Correspondence to: Dr Jun Peng, Academy of Integrative Medicine, Fujian University of Traditional Chinese Medicine, 1 Huatuo Road, Minhou Shangjie, Fuzhou, Fujian 350122, P.R. China

E-mail: pjunlab@hotmail.com

*Contributed equally

Abbreviations: EEPS, ethanol extract of Patrinia scabiosaefolia; CRC, colorectal cancer; IHS, immunohistochemical staining; VEGF-A, vascular endothelial growth factor A; MVD, microvessel density

Key words: Patrinia scabiosaefolia, traditional Chinese medicine, colorectal cancer, angiogenesis

\section{Introduction}

Drug resistance and toxicity to normal cells limit the effectiveness of currently used cancer chemotherapies (1-3), increasing the necessity for the development of new antitumor agents. Natural products, including traditional Chinese medicine (TCM), has recently received great interest since these agents have relatively few side-effects and have been used as important alternative cancer remedies for many years $(4,5)$. Patrinia scabiosaefolia is a perennial plant natively distributed in Eastern Asia. As a well-known Oriental folk medicine, it has long been used in China for the treatment of edema, appendicitis, endometritis and other inflammatory illnesses (6). Patrinia scabiosaefolia is also used as an important component in several TCM formulas to clinically treat cancers $(7,8)$. However, the precise mechanism of its antitumor activity remains largely unknown.

Angiogenesis is a physiological process involving the growth of new blood vessels from the pre-existing vasculature. Although angiogenesis plays an essential role in a wide range of physiological processes such as wound healing, reproduction and embryonic development, deregulation of this vital biological process is strongly associated with cancer progression (9-13). In the initial stage, tumor cells obtain oxygen and nutrients from nearby blood vessels by simple passive diffusion. However, when a tumor grows to reach a certain size, $>2 \mathrm{~mm}^{3}$, oxygen delivery by diffusion is no longer sufficient, causing tumor cells to induce the sprouting of new blood vessels from pre-existing vasculature, creating a blood supply system within the tumor that is essential for continue growth of the tumor as well as providing an avenue for hematogenous metastasis (14-17). Induction of angiogenesis is mediated by a variety of molecules released by tumor cells (18-20), including vascular endothelial growth factor A (VEGF-A) which is considered as one of the strongest stimulators of angiogenesis (21-23). VEGF-A is commonly overexpressed in many types of human cancer, and is correlated with tumor progression, invasion and metastasis, and poorer survival and prognosis in patients (24-27). VEGF-A exerts its biologic effect primarily through interaction with specific receptors present on the surface of vascular endothelial cells (ECs), which in turn triggers a tyrosine kinase signaling cascade, 
inducing EC proliferation, migration, survival, sprouting and eventually tube formation $(21,24,27,28)$. Due to the key role of angiogenesis in the progression of solid tumors, inhibition of tumor angiogenesis has become a major focus of anticancer drug development.

To elucidate the mechanism of the tumorcidal activity of Patrinia scabiosaefolia, using a colorectal cancer (CRC) mouse xenograft model, the human colon carcinoma cell line HT-29 and human umbilical vein endothelial cells (HUVECs), in this study we evaluated its effect on tumor angiogenesis in vivo and in vitro, and investigated the underlying molecular mechanisms.

\section{Materials and methods}

Materials and reagents. Roswell Park Memorial Institute (RPMI)-1640 medium, Dulbecco's modified Eagle's medium (DMEM), fetal bovine serum (FBS), penicillinstreptomycin, Trypsin-EDTA, and TRIzol reagent were purchased from Invitrogen Life Technologies (Carlsbad, CA, USA). SuperScript II reverse transcriptase was obtained from Promega Corporation (Madison, WI, USA). CD31 and VEGF-A antibodies, and secondary antibodies were obtained from Cell Signaling Technology, Inc. (Beverly, MA, USA). The In vitro angiogenesis assay kit was purchased from Millipore (Billerica, MA, USA). The Human VEGF-A ELISA kit was obtained from Shanghai Xitang Biological Technology Ltd. (Shanghai, China). All other chemicals, unless otherwise stated, were obtained from Sigma-Aldrich (St. Louis, MO, USA).

Preparation of ethanol extract from Patrinia scabiosaefolia. EEPS was prepared as described previously (8). For animal experiments, EEPS powder was dissolved in saline to a working concentration of $250 \mathrm{mg} / \mathrm{ml}$. In cell-based experiments, EEPS powder was dissolved in 50\% DMSO to a stock concentration of $250 \mathrm{mg} / \mathrm{ml}$ and the working concentrations of EEPS were made by diluting the stock solution in the culture medium. The final concentration of DMSO in the medium for all cell experiments was $<0.5 \%$.

Cell culture. Human colon carcinoma HT-29 cells and HUVECs were obtained from the Cell Bank of the Chinese Academy of Science (Shanghai, China). HUVECs or HT-29 cells were grown in RPMI-1640 or DMEM, respectively. RPMI-1640 and DMEM were supplemented with $10 \%(\mathrm{v} / \mathrm{v})$ FBS, and $100 \mathrm{U} / \mathrm{ml}$ penicillin and $100 \mu \mathrm{g} / \mathrm{ml}$ streptomycin. Cells were cultured at $37^{\circ} \mathrm{C}$ humidified incubator with $5 \% \mathrm{CO}_{2}$.

Animals. Male BALB/c athymic (nude) mice (with an initial body weight of 20-22 g) were obtained from the Shanghai SLAC Laboratory Animal Co., Ltd. (Shanghai, China) and housed under pathogen-free conditions with controlled temperature $\left(22^{\circ} \mathrm{C}\right)$, humidity and a 12-h light/dark cycle. Food and water were given ad libitum throughout the experiment. All animal treatments were strictly in accordance with international ethical guidelines and the National Institutes of Health Guide concerning the Care and Use of Laboratory Animals, and the experiments were approved by the Institutional Animal Care and Use Committee of Fujian University of Traditional Chinese Medicine.
In vivo tumor xenograft study. HT-29 cells $\left(1.5 \times 10^{6}\right)$ mixed with Matrigel (1:1) were subcutaneously injected into the right flank of the mice to initiate tumor growth. After 5 days of xenograft implantation, mice were randomly divided into 2 groups $(n=6)$ and given intragastric administration with $1.93 \mathrm{~g} / \mathrm{kg} /$ day of EEPS or saline daily, 5 days a week for 21 days. Tumor size was determined by measuring the major (L) and minor (W) diameter with a caliper. The tumor volume was calculated according to the following formula: tumor volume $=\pi / 6 \times \mathrm{L} \mathrm{x} \mathrm{W}^{2}$.

Immunohistochemical staining. After being fixed with $10 \%$ formaldehyde for $12 \mathrm{~h}$, tumor samples were processed conventionally for paraffin-embedded tumor slides. The slides were subjected to antigen retrieval, and the endogenous peroxidase activity was quenched with hydrogen peroxide. After blocking non-specific proteins with normal serum in phosphate-buffered saline (PBS) (0.1\% Tween-20), slides were incubated with rabbit polyclonal antibodies against CD31 and VEGF-A (1:200 dilution). After washing with PBS, slides were incubated with a biotinylated secondary antibody followed by conjugated horseradish peroxidase (HRP)-labeled streptavidin (Dako), and then washed with PBS. The slides were then incubated with diaminobenzidine (DAB) (Sigma) as the chromogen, followed by counterstaining with diluted Harris' hematoxylin (Sigma). After staining, 5 high-power fields (x400) were randomly selected in each slide, and the average proportion of positive cells in each field was counted using a true color multi-functional cell image analysis management system (Image-Pro Plus; Media Cybernetics, Rockville, MD, USA). To rule out any nonspecific staining, PBS was used to replace the primary antibody as a negative control.

Evaluation of cell viability by MTT assay. Viability of HUVECS was assessed by the 3-(4,5-dimethylthiazol-2-yl)2,5-diphenyltetrazolium bromide (MTT) colorimetric assay. HUVECs were seeded into 96-well plates at a density of $1 \times 10^{4}$ cells/well in $0.1 \mathrm{ml}$ medium. The cells were treated with various concentrations of EEPS for 24, 48 and $72 \mathrm{~h}$ respectively. At the end of the treatment, $100 \mu 1$ MTT $(0.5 \mathrm{mg} / \mathrm{ml}$ in PBS) was added to each well, and the samples were incubated for an additional $4 \mathrm{~h}$ at $37^{\circ} \mathrm{C}$. The purple-blue MTT formazan precipitate was dissolved in $100 \mu \mathrm{l}$ DMSO. The absorbance was measured at $570 \mathrm{~nm}$ using an ELISA reader (BioTek, Model ELX800; BioTek Instruments, Inc., Winooski, VT, USA).

Migration assay of HUVECs. Migration of HUVECs was performed by the wound healing method. HUVECs were seeded into 12 -well plates at a density of $2 \times 10^{5}$ cells/well in $1 \mathrm{ml}$ medium. After $24 \mathrm{~h}$ of incubation, cells were scraped away vertically in each well using a P100 pipette tip. Three randomly selected views along the scraped line were photographed for each well using a phase-contrast inverted microscope at a magnification of x100. Cells were then treated with the indicated concentrations of EEPS for $24 \mathrm{~h}$, and another set of images was captured using the same method. A reduction in the scraped area indicated migratory capability.

Tube formation assay of HUVECs. The tube formation of HUVECs was examined using the ECMatrix assay kit 


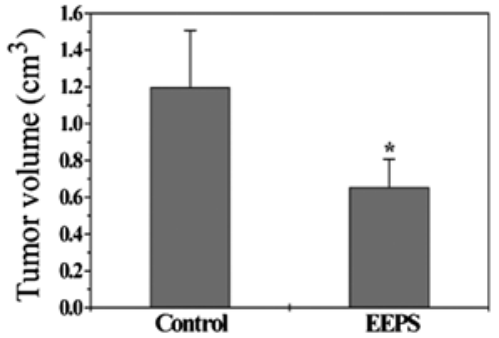

Figure 1. Effect of EEPS on tumor growth in CRC xenograft mice. After tumor development, the mice were given intragastric administration of $1.93 \mathrm{~g} / \mathrm{kg}$ of EEPS daily, 5 days a week for 21 days. Tumor volume was measured at the end of the experiment. Data shown are averages with SD (error bars) from 6 individual mice in each group. ${ }^{*} \mathrm{P}<0.05$, vs. controls. EEPS, ethanol extract of Patrinia scabiosaefolia; CRC, colorectal cancer

(Millipore), following the manufacturer's instructions. Briefly, confluent HUVECs were harvested and diluted $\left(1 \times 10^{4}\right.$ cells) in $50 \mu 1$ of medium, containing indicated concentrations of EEPS. The harvested cells were then seeded into 1:1 ECMatrix gel (v/v)-coated 96 -well plates and incubated for $9 \mathrm{~h}$ at $37^{\circ} \mathrm{C}$. The network-like structures were examined using a phase-contrast inverted microscope. The images were captured at a magnification of $x 100$. The level of tube formation was quantified by calculating the length of tubes in 3 randomly chosen fields from each well.

Measurement of VEGF-A secretion in HT-29 cells by ELISA. HT-29 cells were seeded into 6-well plates at a density of $2 \times 10^{5}$ cells/well in $2 \mathrm{ml}$ medium and treated with the indicated concentrations of EEPS for $24 \mathrm{~h}$. The level of VEGF-A in the media was measured using an ELISA kit (XiTang Biological Technology Ltd., Shanghai, China) according to the manufacturer's instructions. The concentration of VEGF-A was determined by comparison to serial dilutions of a purified standard of VEGF-A.

RT-PCR analysis. Total RNA was isolated from tumor tissues or HT-29 cells with TRIzol reagent. Oligo(dT)-primed RNA $(1 \mu \mathrm{g})$ was reverse-transcribed with SuperScript II reverse transcriptase (Promega Corporation) according to the manufacturer's instructions. The obtained cDNA was used to determine the mRNA amount of VEGF-A by PCR with Taq DNA polymerase (Fermentas). GAPDH was used as an internal control.

Statistical analysis. Data are presented as means \pm SD for the indicated number of independently performed experiments and were analyzed using the SPSS Package for Windows (version 16.0). Statistical analysis of the data was performed with the Student's t-test and ANOVA. Differences with $\mathrm{P}<0.05$ were considered to be statistically significant.

\section{Results and Discussion}

EEPS inhibits tumor growth in colorectal cancer (CRC) xenograft mice. We evaluated the tumor growth in CRC xenograft mice by measuring the tumor volume. Data in Fig. 1 indicate that EEPS treatment significantly suppressed tumor growth, as compared with the control group. The final tumor

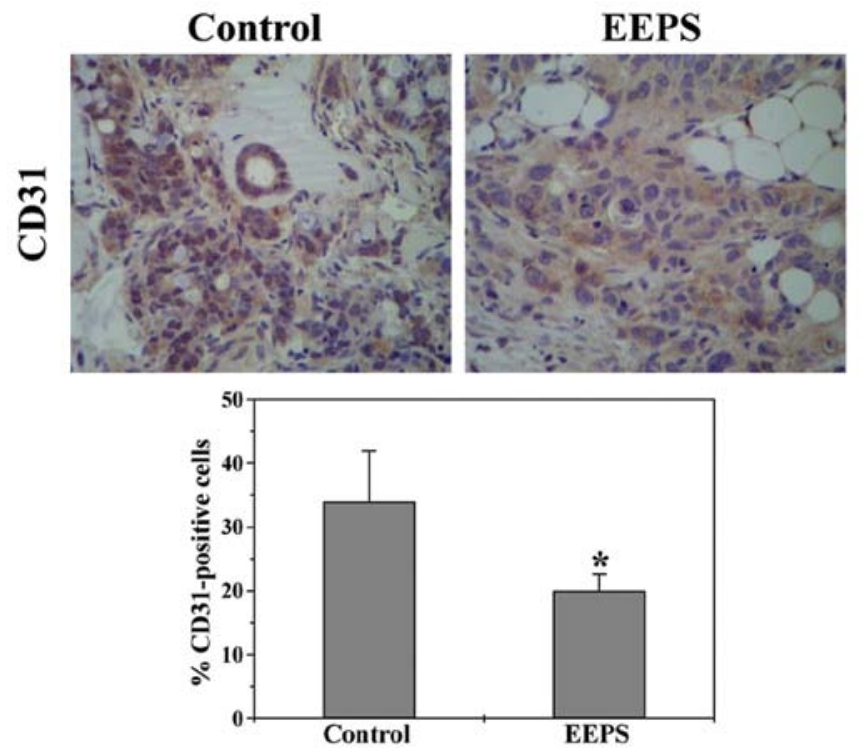

Figure 2. Effect of EEPS on the intratumoral microvessel density in CRC xenograft mouse tumors. Tumor tissues were processed for immunohistochemical staining (IHS) for CD31. The photographs are representative images captured at a magnification of $x 400$. Quantification of IHS assay is represented as the percentage of positively stained cells. Data shown are averages with SD (error bars) from 6 individual mice in each group. "P<0.05, vs. controls. EEPS, ethanol extract of Patrinia scabiosaefolia; CRC, colorectal cancer.

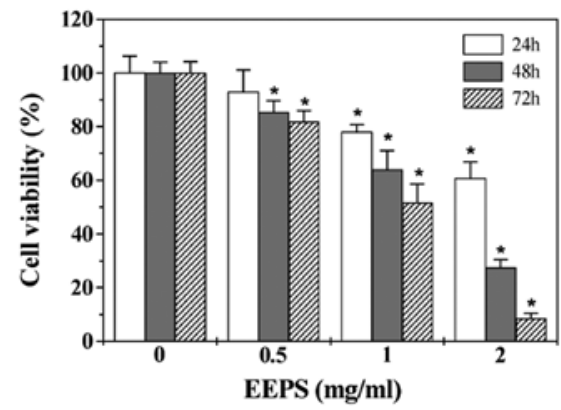

Figure 3. Effect of EEPS on the viability of HUVECs. Cell viability was determined by the MTT assay after HUVECs were treated with various concentrations of EEPS for 24, 48 or $72 \mathrm{~h}$. The data were normalized to the viability of the control cells (100\%). Data are averages with SD (error bars) from 3 independent experiments. ${ }^{*} \mathrm{P}<0.05$, vs. control cells. EEPS, ethanol extract of Patrinia scabiosaefolia; HUVECs, human umbilical vein endothelial cells.

volume/mouse in the control and the EEPS-treated group was $1.20 \pm 0.31$ and $0.65 \pm 0.15 \mathrm{~cm}^{3}$, respectively $(\mathrm{P}<0.05)$, suggesting that EEPS was effective in the treatment of colorectal cancer.

EEPS displays anti-angiogenic activity in CRC xenograft mouse tumors and in human umbilical vein endothelial cells. Angiogenesis plays an important role in the progression and metastasis of cancer, which thus has become an attractive target for anticancer therapy. The expression of endothelial cell-specific marker CD31 in CRC tumor tissues was determined by immunohistochemical staining (IHS) to evaluate the effect of EEPS on intratumoral microvessel density (MVD), an indicator of new vessel growth. As shown in Fig. 2, the percentage of CD31-positive cells in the EEPS-treated mouse 

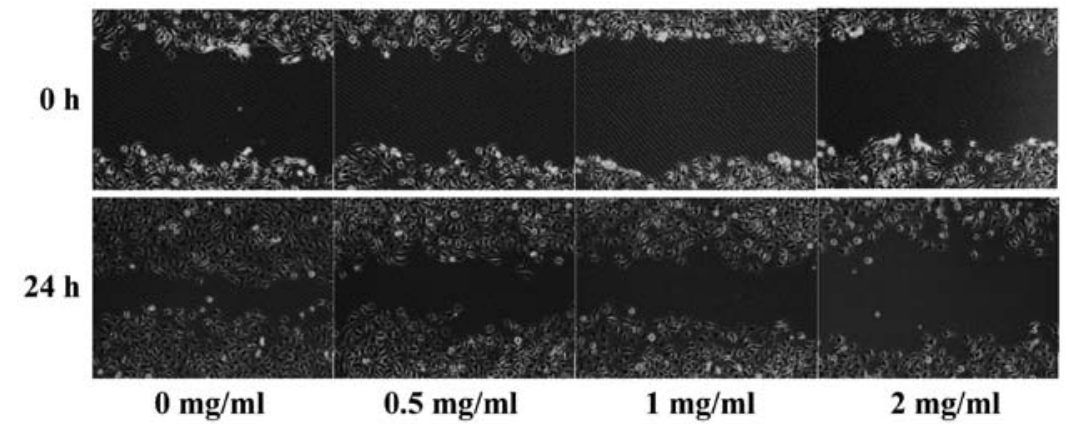

Figure 4. Effect of EEPS on the migration of HUVECs. After treatment with the indicated concentrations of EEPS for $24 \mathrm{~h}$, the migration pattern of HUVECs was observed using phase-contrast microscopy. The photographs were captured at a magnification of x100. Images are representative of 3 independent experiments. EEPS, ethanol extract of Patrinia scabiosaefolia; HUVECs, human umbilical vein endothelial cells.
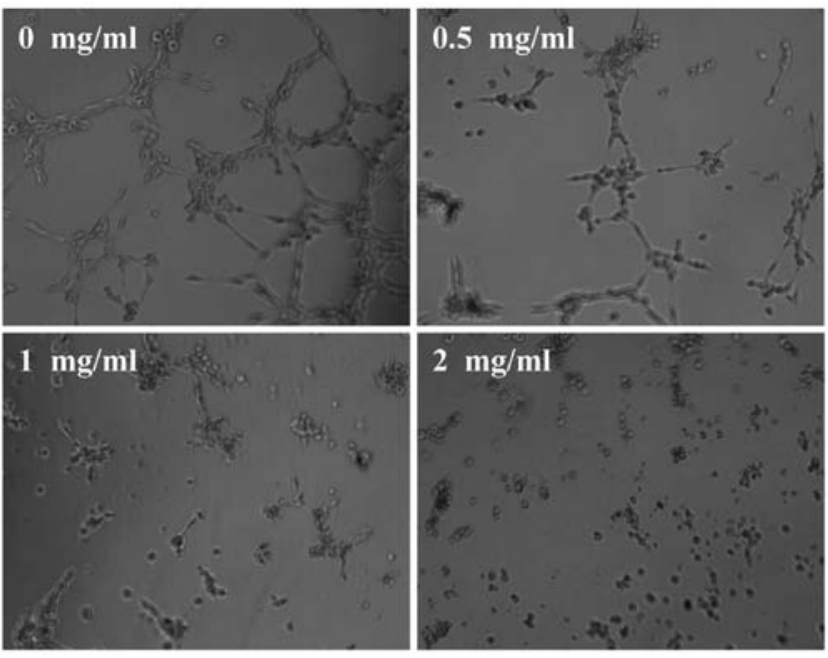

Figure 5. Effect of EEPS on HUVEC tube formation. HUVECs were harvested and diluted in medium containing various concentrations of EEPS. The harvested cells were then seeded into 1:1 ECMatrix gel (v/v)-coated plates and incubated for $9 \mathrm{~h}$ at $37^{\circ} \mathrm{C}$. The network-like structures were examined using phase-contrast microscopy. The photographs were captured at a magnification of x100. Images are representative of 3 independent experiments. EEPS, ethanol extract of Patrinia scabiosaefolia; HUVECs, human umbilical vein endothelial cells.

A

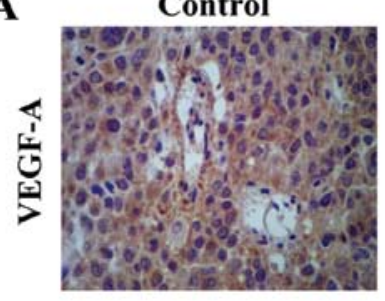

B

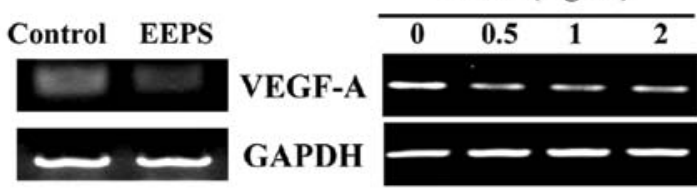

EEPS

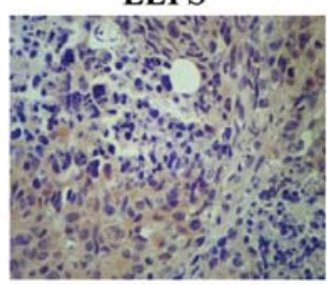

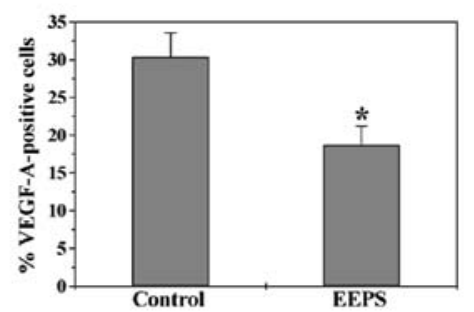

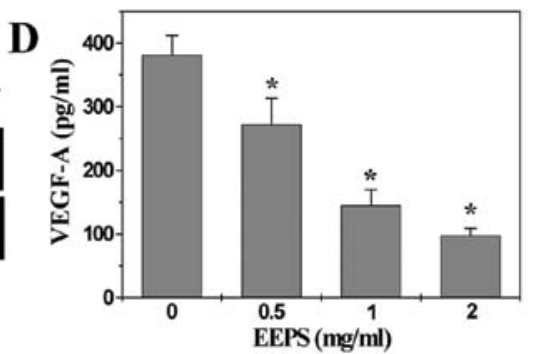

Figure 6. Effect of EEPS on the expression of VEGF-A in CRC xenograft mouse tumors and HT-29 cells. (A) Tumor tissues were processed for IHS for VEGF-A. The photographs are representative images captured at a magnification of x400. Quantification of IHS assay is represented as the percentage of positively stained cells. Data shown are averages with SD (error bars) from 6 individual mice in each group. The mRNA level of VEGF-A in (B) tumor tissues or in (C) HT-29 cells was determined by RT-PCR. GAPDH was used as the internal control. Images are representatives of 6 individual mice in each group or 3 independent cell-based experiments. (D) The protein secretion level of VEGF-A was determined by ELISA in HT-29 cells. Data are averages with SD (error bars) from 3 independent experiments. "P<0.05, vs. controls. EEPS, ethanol extract of Patrinia scabiosaefolia; VEGF-A, vascular endothelial growth factor A; CRC, colorectal cancer; IHS, immunohistochemical staining. 
tumors was significantly reduced $(\mathrm{P}<0.05)$, demonstrating the in vivo anti-angiogenic activity of EEPS.

Angiogenesis typically consists of several features, including proliferation, migration and eventually the capillary tube formation of endothelial cells (ECs). To further evaluate the anti-angiogenic effect of EEPS we modeled each of these processes with HUVECs in vitro. As shown in Fig. 3, EEPS treatment dose- and time-dependently decreased the proliferation (viability) of HUVECs $(\mathrm{P}<0.05$, compared to untreated control cells). Moreover, EEPS treatment inhibited HUVEC migration after monolayer wounding (Fig. 4). Furthermore, we examined the effect of EEPS on capillary tube formation of HUVECs using an extracellular matrix. As shown in Fig. 5, untreated HUVECs formed elongated tube-like structures; which, however, was profoundly inhibited by EEPS treatment. Taken together, it is suggested that the antitumor angiogenesis activity of EEPS could have contributed to its inhibitory effect on colorectal tumor growth.

EEPS decreases VEGF-A expression in CRC xenograft mouse tumors and human colon carcinoma HT-29 cells. The process of tumor angiogenesis is tightly regulated by a variety of molecules released by tumor cells. Vascular endothelial growth factor A (VEGF-A) is considered as one of the most potent angiogenic stimulators. Overexpression of VEGF-A is found in numerous types of cancer and commonly suggests poor prognosis. VEGF-A functions primarily via a paracrine pathway, binding to its specific receptors located on vascular ECs after secretion from tumor cells, leading to a series of angiogenic processes. To further investigate the mechanism of the anti-angiogenic activity of EEPS, we examined its effect on the expression of VEGF-A. As shown in Fig. 6, EEPS treatment obviously decreased the mRNA and protein expression levels of VEGF-A both in CRC tumor tissues and in HT-29 cells $(\mathrm{P}<0.01)$.

In conclusion, here for the first time we demonstrated that Patrinia scabiosaefolia inhibits colorectal cancer growth via inhibition of tumor angiogenesis, which may in part explain its anticancer activity.

\section{Acknowledgements}

This study was sponsored by the National Natural Science Foundations of China (nos. 81173272 and 81073097).

\section{References}

1. Gorlick R and Bertino JR: Drug resistance in colon cancer. Semin Oncol 26: 606-611, 1999.

2. Boose G and Stopper H: Genotoxicity of several clinically used topoisomerase II inhibitors. Toxicol Lett 116: 7-16, 2000

3. Longley DB, Allen WL and Johnston PG: Drug resistance, predictive markers and pharmacogenomics in colorectal cancer. Biochim Biophys Acta 1766: 184-196, 2006.

4. Newman DJ, Cragg GM and Snader KM: The influence of natural products upon drug discovery. Nat Prod Rep 17: 215-234, 2000.
5. Gordaliza M: Natural products as leads to anticancer drugs. Clin Transl Oncol 9: 767-776, 2007.

6. Ju HK, Baek SH, An RB, Bae K, Son KH, Kim HP, Kang SS Lee SH, Son JK and Chang HW: Inhibitory effects of nardostachin on nitric oxide, prostaglandin E2, and tumor necrosis factor- $\alpha$ production in lipopolysaccharide-activated macrophages. Biol Pharm Bull 26: 1375-1378, 2003.

7. Chiu LC, Ho TS, Wong EY and Ooi VE: Ethyl acetate extract of Patrinia scabiosaefolia downregulates anti-apoptotic $\mathrm{Bcl}-2 / \mathrm{Bcl}-\mathrm{X}(\mathrm{L})$ expression, and induces apoptosis in human breast carcinoma MCF-7 cells independent of caspase-9 activation. J Ethnopharmacol 105: 263-268, 2006.

8. Peng J, Chen YQ, Lin JM, Zhuang ZQ, Xu W, Hong ZF and Sferra TJ: Patrinia scabiosaefolia extract suppresses proliferation and promotes apoptosis by inhibiting the STAT3 pathway in human multiple myeloma cells. Mol Med Rep 4: 313-318, 2011.

9. Folkman J: Tumor angiogenesis: therapeutic implications. N Engl J Med 285: 1182-1186, 1971.

10. Folkman J and Shing Y: Angiogenesis. J Biol Chem 267: 10931-10934, 1992.

11. Folkman J: Angiogenesis in cancer, vascular, rheumatoid and other disease. Nat Med 1: 27-31, 1995.

12. Folkman J: Angiogenesis. Annu Rev Med 57: 1-18, 2006.

13. Cook KM and Figg WD: Angiogenesis inhibitors: current strategies and future prospects. CA Cancer J Clin 60: 222-243, 2010.

14. Mantovani A, Allavena P, Sica A and Balkwill F: Cancer-related inflammation. Nature 454: 436-444, 2008.

15. Whiteside TL: The tumor microenvironment and its role in promoting tumor growth. Oncogene 27: 5904-5912, 2008.

16. Jain RK: Transport of molecules in the tumor interstitium: a review. Cancer Res 47: 3039-3051, 1987.

17. Folkman J: How is blood vessel growth regulated in normal and neoplastic tissue? G.H.A. Clowes memorial Award lecture. Cancer Res 46: 467-473, 1986.

18. Strömblad S and Cheresh DA: Integrins, angiogenesis and vascular cell survival. Chem Biol 3: 881-885, 1996.

19. Breier G and Risau W: The role of vascular endothelial growth factor in blood vessel formation. Trends Cell Biol 6: 454-456, 1996.

20. Weidner N, Semple JP, Welch WR and Folkman J: Tumor angiogenesis and metastasis - correlation in invasive breast carcinoma. N Engl J Med 324: 1-8, 1991.

21. Ferrara N: Role of vascular endothelial growth factor in physiologic and pathologic angiogenesis: therapeutic implications. Semin Oncol 29: 10-14, 2002.

22. Jain RK: Tumor angiogenesis and accessibility: role of vascular endothelial growth factor. Semin Oncol 29: 3-9, 2002.

23. Risau W: Mechanisms of angiogenesis. Nature 386: 671-674, 1997.

24. Ferrara N, Gerber HP and LeCouter J: The biology of VEGF and its receptors. Nat Med 9: 669-676, 2003.

25. Kaya M, Wada T, Akatsuka T, Kawaguchi S, Nagoya S, Shindoh M, Higashino F, Mezawa F, Okada F and Ishii S: Vascular endothelial growth factor expression in untreated osteosarcoma is predictive of pulmonary metastasis and poor prognosis. Clin Cancer Res 6: 572-577, 2000.

26. Maeda K, Chung YS, Ogawa Y, Takatsuka S, Kang SM, Ogawa M, Sawada T and Sowa M: Prognostic value of vascular endothelial growth factor expression in gastric carcinoma. Cancer 77: 858-863, 1996.

27. Ishigami SI, Arii S, Furutani M, Niwano M, Harada T, Mizumoto $\mathrm{M}$, Mori A, Onodera $\mathrm{H}$ and Imamura $\mathrm{M}$ : Predictive value of vascular endothelial growth factor (VEGF) in metastasis and prognosis of human colorectal cancer. Br J Cancer 78: 1379-1384, 1998.

28. Gille H, Kowalski J, Li B, LeCouter J, Moffat B, Zioncheck TF, Pelletier N and Ferrara N: Analysis of biological effects and signaling properties of Flt-1 (VEGFR-1) and KDR (VEGFR-2). A reassessment using novel receptor-specific vascular endothelial growth factor mutants. J Biol Chem 276: 3222-3230, 2001. 